\title{
A Prototype of Co-Frequency Co-Time Full Duplex Networking
}

\author{
Meng Ma, Shuyi Tian, Yingyang Chen, Li Wang, Yuli Yang, Lei Wan, Bingli Jiao, and H. Vincent Poor
}

\begin{abstract}
Radio full duplex (FD) has emerged as an attractive technique capable of doubling the spectral efficiency over half duplex. However, for signal reception, an FD node needs to suppress its transmitter's signals quite significantly. In point to point communication systems, these transmitter's signals are termed self-interference. When working with an FD mobile network, the self-interference problem becomes much more complicated because the receiver of an FD base station (BS) receives interference not only from its BS transmitter in its cell, but also from those in the surrounding cells. For the uplink (UL) channel, self-interference extends to the problem of multiple interference. And, a similar interference problem can be found among the mobile stations (MSs) over a downlink (DL) channel. In both cases, the interference owing to the FD implementation spreads beyond the scope of the self-interference. This paper describes the development of FD BSs that use antenna arrays to deal with the BSs' interference and, thus, enable FD communication over the UL channel, where the theoretical focus is placed on how to use the antenna array to nullify the multiple interference and receive the signals of the desired MSs simultaneously. To complete the system construction, FD MSs have also been developed to enable DL transmission. A prototype system is described for the scenario of two cells and one FD MS for tests of FD communication over UL- and DL channels in terms of video performance. Good video quality is demonstrated at both the BS and MS.
\end{abstract}

Index Terms-Full Duplex, Networking, Antenna Array

\section{INTRODUCTION}

Mobile systems are experiencing dramatic increases in the demand for data capacity, and this trend is expected to continue well into the future. One of the crucial challenges caused by this demand is the scarcity of radio frequency (RF) spectrum below $6 \mathrm{GHz}$ that can provide good coverage. Thus, developing radio full duplex (FD) is of great interest due to

M. Ma, S. Tian, Y. Chen, and B. Jiao are with the School of Electronics Engineering and Computer Science, Peking University, Beijing 100871, China (email: $\{$ mam, tianshuyi, chenyingyang, jiaobl\}@pku.edu.cn). (Corresponding author: Bingli Jiao.)

L. Wang is with the School of Electronic Engineering, Beijing University of Posts and Telecommunications, Beijing 100876, China. She is also with the Key Laboratory of the Universal Wireless Communications, Ministry of Education, P.R.China. (e-mail: liwang@bupt.edu.cn).

Y. Yang is with the Department of Electronic and Electrical Engineering, University of Chester, Chester CH2 4NU, U.K. (e-mail: y.yang@chester.ac.uk).

L. Wan is with Huawei Technologies Company Ltd., Beijing 100085, China (e-mail: wan.lei@huawei.com).

H. V. Poor is with the Department of Electrical Engineering, Princeton University, Princeton, NJ 08544 USA (e-mail: poor@princeton.edu).

This work was jointly supported by National Natural Science Foundation of China under Grants No. 61531004, No. 61871416, and the Beijing Municipal Natural Science Foundation under Grant L172010. its capability for doubling, potentially, the spectral efficiency of either frequency division duplex or time division duplex by allocating transmit- and receive signals in co-frequency and co-time $[1,2]$.

Critical to the implementation of the FD technique, in a practical system, is the capability of canceling the selfinterference that comes from the transmitter to the receiver [2]. Techniques for self-interference cancellations are reviewed in [3]. Advanced self-interference cancellation allows bidirectional communication over 500 meters in a point to point (P2P) communication system. These advanced techniques also enables FD's applications in cognitive radios [4] and cooperative communications [5]. Moreover, combining multiple input multiple output (MIMO) with FD has been proposed for exploiting spatial diversity [6].

However, when the application of FD evolves into mobile networking, the simple topology of P2P communication transforms itself to a configuration of multiple FD nodes, whereby the self-interference presents itself to all. For example, all base station (BS) receivers can suffer from the interference not only from their own BS transmitters, but also from those in their neighboring cells, especially when the entire spectrum is used $[7,8]$. Thus, with techniques developed for a single FD node, reliable communication is difficult to achieve in mobile networking scenario. To push FD to mobile networking, it is needed to develop a systematic approach encompassing multiple elements for dealing with the interference. As far as FD networking is concerned, there is a paucity of literature on prototype-demonstration.

Hence, in this paper, we provide a networking solution that allows all BSs and MSs working in the manner of co-frequency co-time full duplex (CCFD) in a cellular structure, and shows the FD functions using a prototype system. Specifically, for the uplink (UL) channel, the strong BS to BS (B2B) interference should be suppressed significantly at every BS receiver. To illustrate this scenario, the $\mathrm{B} 2 \mathrm{~B}$ interference is shown on the top of Fig. 1 for implementing an FD BS in a conventional manner with a cellular structure. While, for the downlink (DL) channel, apart from the self-interference, we need to consider the possible interference among the mobile stations (MSs). To differentiate this, we use BS to BS (B2B) interference and MS to MS (M2M) interference to replace the self-interference for the expressions of UL and DL channels, respectively. In addition, the term full duplex interference will be used to include B2B and M2M interference as well. 
To cope with the B2B interference, we present a new design that allocates a single transmit antenna in the center of, and three BS receive antenna arrays distributed inside, each cell. The left corner in the bottom of Fig. 1 shows the antenna configuration of a cell. The role of each BS receive antenna array is to perform a receiving beam towards the desired MS and reject all B2B interference (including the interference delivered from its own BS transmitter) simultaneously as shown in the bottom right corner. The advantages of this design are three-fold. First, the B2B interference inside the cell can be dramatically attenuated due to the path loss over the distance from the BS transmit antenna to the receive antenna array. Second, the antenna array of the BS receiver can employ the beamforming to reject the B2B interference not only from its own cell but also from neighboring cells. The third lies in the gain factor of the antenna array that can increase the signal to interference plus noise ratio (SINR) of the desired MS at the BS receiver, and also accelerate the convergence of beamforming [9]. To obtain the secondand third advantages mentioned above simultaneously, we require that the number of array elements should be larger than the number of interfering BSs that are considered. Normally, cluster sizes of 7, 11 or 19 can be taken as the number of interfering BSs.

The third advantage can also alleviate the self-inference problem of the FD MS in the DL channel, because the high gain factor of the array allows a low power transmission and the self-interference will be smaller than the situation without the array gain. Consequently, the requirement of selfinterference cancellation is reduced at the MS.

Using all the advantages mentioned above, we have constructed a prototype system and demonstrated it at the Forum of the Next Generation Mobile Network (NGMN) in March of 2016. The prototype system works very well for video transmissions in two cells, each of which covers 15 meters in radius. Although the experiments consider only a miniature cell application, we believe that the progress from the prototype to practical networking is possible and, thus, these experiments bring the prospect of approximately doubling the spectral efficiency of practical systems, and hence doubling the value of spectrum assets.

In the rest of the paper, we present the details as follows. The main design principle is presented in Section II, and the system components and the specifications are described in Section III. The prototype system is described in Section IV, and the test results are given in Section V, with conclusions and future directions given in Section VI.

\section{Design PRinciple And ANALysis}

In cellular systems, the general-purpose of the communication is to transmit the BS's signal to the desired MS receiver and the MS's signal back to the BS receiver. However, the transmitted signals can become interference when they arrive at any undesired receiver. Since wireless communication is
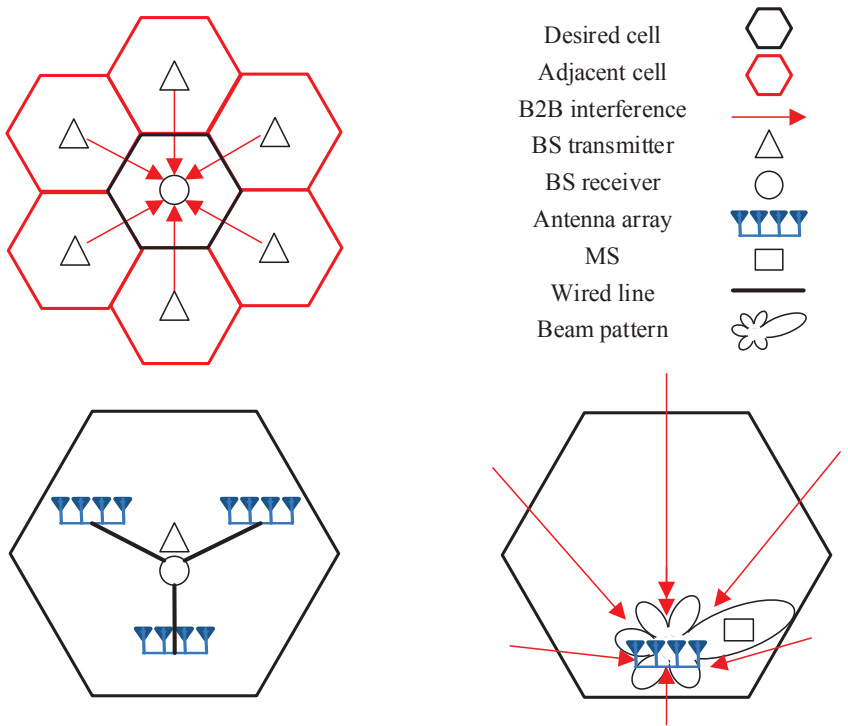

Figure 1. Proposed CCFD networking.

carried out over the air, the interference problem is inevitable and becomes more severe when the system employs an entire spectrum allocation.

In contrast to conventional half-duplex systems, the implementation of CCFD to networking can bring two additional types of interference as listed below.

- B2B interference: each BS receiver is exposed to all BSs' transmitted signals when it is receiving the desired signal from the MS on the UL channel. The BS-transmit-signals belong to the $\mathrm{B} 2 \mathrm{~B}$ interference in the CCFD system.

- M2M interference: when an MS receives its desired signals over the DL channel, it can suffer from the interference delivered from its transmitter and those from other MSs in its surroundings. This is the M2M interference.

These two types of interference are the duplex interference defined in the above section.

Without losing generality, we take a desired cell BS for consideration of the $\mathrm{B} 2 \mathrm{~B}$ interference problem inside the cluster as shown in the bottom right corner of Fig. 1. To suppress this interference, i.e., B2B interference, we arrange that the BSs and FD MS transmit their training sequences in an orthogonal manner to enable beamforming in the following two steps.

At the first step, all BSs transmit synchronously their mutually orthogonal training sequences, which are used by each BS receive array of $M$ antennas to implement the zero forcing method as

$$
\mathbf{w}^{H} \mathbf{A}=\mathbf{0}_{N \times 1}
$$

with $M>N$, where $M$ and $N$ are the numbers of antenna array elements and interfering BSs. $\mathbf{w}$ is the weight vector, and $\mathbf{A}$ is an $M \times N$ steering matrix, where the $i$ th column is the steering vector of the $i$ th interfering $\mathrm{BS}$, which can 
be estimated by using the training sequence. " $H$ " represents the conjugate transpose. In $M$-dimensional signal space, one can always find at least $M-N$ orthogonal bases satisfying the $N$ orthogonality conditions in (1). We denote the bases orthogonal to each volume of $A$ by an $(M-N) \times M$ matrix $\mathbf{W}_{\mathbf{0}}$. By utilizing the $M-N$ degrees of freedom in the subspace $\mathbf{W}_{\mathbf{0}}$, we can further optimize the weights to minimize the signal distortion and noise. In the second step, we project the received signal vector $\mathbf{x}(k)$ onto the $M-N$ bases to obtain a signal vector $\mathbf{x}_{\mathbf{0}}(k)=\mathbf{W}_{\mathbf{0}} \mathbf{x}(k)$, and then minimize following cost function:

$$
J(\mathbf{w})=\left|\mathbf{w}_{F}^{H} \mathbf{x}_{\mathbf{0}}(k)-d(k)\right|^{2}
$$

where $d(k)$ is the $k$ th symbol of the training sequence of the desired MS. A standard adaptive algorithm can be used to minimize (2) by searching for the optimum solution, e.g., Least Mean Squares (LMS) or Recursive Least Squares (RLS). Finally, the antenna array's weight vector can be obtained by multiplying the solutions of the first and second steps, i.e., $\mathbf{w}=\mathbf{W}_{\mathbf{0}}{ }^{H} \mathbf{w}_{F}$.

To conclude the combination of the two step beamforming, we note that the first step of zero forcing is very important due to its ability to avoid the strong B2B interference in the beamforming of the second step. Actually, the adaptive algorithm, whether LMS or RLS, can start its beamforming with little influence of the B2B interference with assistance of the zero forcing in the first step and, thus, converges much faster in comparison with that of merely working with the second step [9].

After completing the whole training procedure of the beamforming, the bi-directional transmission is initiated from the BS to the desired MS and from the MS to the BS simultaneously.

In this design, there is no intra-cell M2M interference because the MSs' signals are orthogonal in the time domain, while the inter-cell M2M interference can be neglected because scheduling can be used to separate the two MSs far apart in distance, whereat the signal actuation plays the role. Finally, the nullification of the self-interference of the FD MS will be explained in the next section.

\section{HARDWARE DESCRIPTION}

To verify the effectiveness of the proposed CCFD networking method, we developed a demo system consisting of two FD BSs and one FD MS by using the WiFi (IEEE 802.11a) specifications (with a modification only on the transmit timing of the training sequence) to perform the bi-directional communication. In contrast to the hardware of $\mathrm{WiFi}$, we added an array processor and self-interference canceler to the receiver of the BS and that of the MS, respectively.

The major hardware implementations are explained as shown in Fig. 2.

\section{A. Hardware Components}

Xilinx ZC706 evaluation board: This is a highperformance evaluation board for the baseband signal processing configured with the Zynq-7000 XC7Z045 All Programmable system-on-chip (AP SoC) which consists of a dual-core $\mathrm{ARM} \otimes$ Cortex $^{\mathrm{TM}}$-A9 MPCore ${ }^{\mathrm{TM}}$ based processing system (PS) and Xilinx programmable logic (PL) in a single device. It also supports a rich set of interfaces to extend features of the board, e.g., the FPGA Mezzanine Card (FMC) connectors [10].

AD9361: This is a high-performance RF transceiver integrated with 12-bit DACs and ADCs. The device combines an RF front end with a flexible mixed-signal baseband section and integrated frequency synthesizers to provide a configurable digital interface to a processor via an FMC connector. In addition, the AD9361 can adapt to a wide-range of the spectrum ranging from $70 \mathrm{MHz}$ to $6.0 \mathrm{GHz}$, and it can support a broadband operation ranging from $200 \mathrm{KHz}$ to $56 \mathrm{MHz}$ [11].

YunSDR Y310: This is a software-defined radio (SDR) platform consisting of an AD9361 and Zynq-7000 XC7Z020 SoC which includes a dual-core ARM@Cortex ${ }^{\mathrm{TM}}-\mathrm{A} 9$ based PS and Xilinx PL as the baseband processing module. The platform also supports the connection to an external reference clock that can be used for synchronization [12].

RF Self-interference canceler: This is developed by the present authors to work as an RF self-interference canceler. The RVA-3000+ and JSPHS-2484+ are used to adjust amplitudes and phases of the RF signals, respectively, via an analog control voltage.

Full duplex antenna: The full duplex antenna consists of one Rx antenna and one Tx antenna arranged geometrically in a straight line which provides, for the receiver, high interference isolation of transmit signals [13].

Antenna array: The antenna array of the BS receiver consists of four whip antennas, each of which has about 5 $\mathrm{dBi}$ power gain at $2.4 \mathrm{GHz}$.

\section{B. System Construction}

The BS transmitter is constructed with the ZC706 and AD9361 as digital- and RF signal processor, respectively. In the BS receiver, the YunSDR Y310 collects RF signals from the antenna array and carries out the tasks of the array processor with assistance of a $\mathrm{PC}$ in signal processing.

The FD MS consists, mainly, of ZC706, AD9361, RF selfinterference canceler and the full duplex antenna. The selfinterference is reduced by the full duplex antenna, RF selfinterference canceler and the digital canceler in a ZC706 step by step.

Finally, to enable the accurate timing of the training sequence transmission, the BSs are synchronized by a clock through a coaxial cable's link.

\section{The Operation of the Prototype}

This section explains the operation of the CCFD networking in the scenario of two cells and one FD MS for bi-directional 


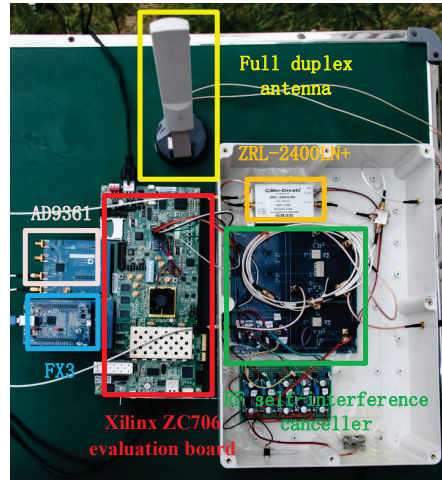

(a)

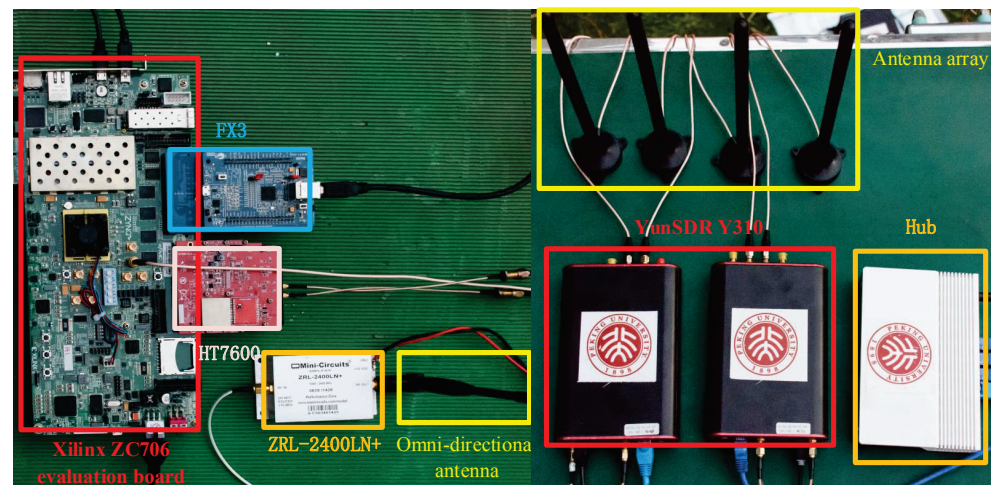

(b)

Figure 2. (a) FD MS hardware components; (b) BS transmitter and BS receiver hardware components.

communication over UL- and DL channels. The bi-directional communication between the BS and MS are described below.

\section{A. Frame Structure Design}

The data frame structure of the transmit signals of both the proposed BS and MS are designed based on IEEE 802.11a physical layer specifications [14][15].

Let us review the IEEE 802.11a for the physical layer, where the data frame can be divided into two parts: the header and the data field. The header can be further divided into the training sequence and SIG as shown at the top of Fig. 3(a).

The WiFi receiver uses the training sequence to perform the time synchronization and frequency offset for enabling the signal reception by reading the useful information, such as channel coding and length of the data field. The signal transmission and the reception need 64 point FFT- and IFFT converters to work with the 64 sub-carriers of OFDM.

The difference between the proposed data frame and that of IEEE 802.11a can be found by comparing the two as shown in Fig. 3(a), where the proposed system arranges the transmission of the training sequences in such a way that they are not overlapped in the time domain. Thus, at the transmitter of the proposed system, each data frame is separated. enable the beamforming of the two steps as explained in Section II. Apart from the timing difference of the training sequences, all other aspects are the same as that of IEEE 802.11a.

In the following subsections, we explain the method for dealing with the full duplex interference.

\section{B. UL Channel}

The full duplex interference over the UL channel is the B2B interference which comes from the DL signal transmissions. For suppressing this interference, the antenna array of the BS receiver receives the training sequences from the transmitter of its own BS and that of the other BS, and performs the first step of beamforming as described in (1) and (2) of Section II. Then, it receives the training sequence transmitted by the FD MS to complete the beam's directivity in the second step.
Finally, the BS receiver is ready for receiving the signals from the MS despite the presence of strong B2B interference.

\section{DL Channel}

For the DL channel, the M2M interference can be, in general, categorized into the intra-cell interference and intercell interference. In this design, the former is found only at the FD MS, i.e., self-interference, because the system can assign each of the MSs a time slot using time division access to avoid the M2M interference among the FD MSs in the cell. In other words, we do not allow any two MSs to transmit their signals simultaneously in the same cell. While, for the inter-cell interference, we can avoid it by allocating the same time slots to the MSs, which are far away from each other. In mobile network system, this belongs to the resource allocation via scheduling procedures.

Thus, we only need to show the performance of one FD MS for its bi-direction communication. Actually, the FD MS starts its self-interference cancellation when its transmitter delivers the training sequence to the BSs and works in the same manner as that of an FD node in the P2P communication system.

\section{Bi-Direction Communication}

Once the beamforming procedures are completed, the CCFD communication begins: the two BS-transmitters and the MS begin their signal transmission simultaneously, while the BS receiver receives the UL channel signals and the MS receives the DL signals. The B2B interference is rejected by the beamforming. The M2M interference, i.e., self-interference, is canceled by the MS's canceler.

\section{E. Prototype Design}

In this subsection, the block structure of the developed CCFD system is explained for the BS transmitter, BS receiver and FD MS as shown in Fig. 3(b). In the BS transmitter, the video bits are fed by the PC host and sent to a ZC706 evaluation board for digital signal processing. These bits are 


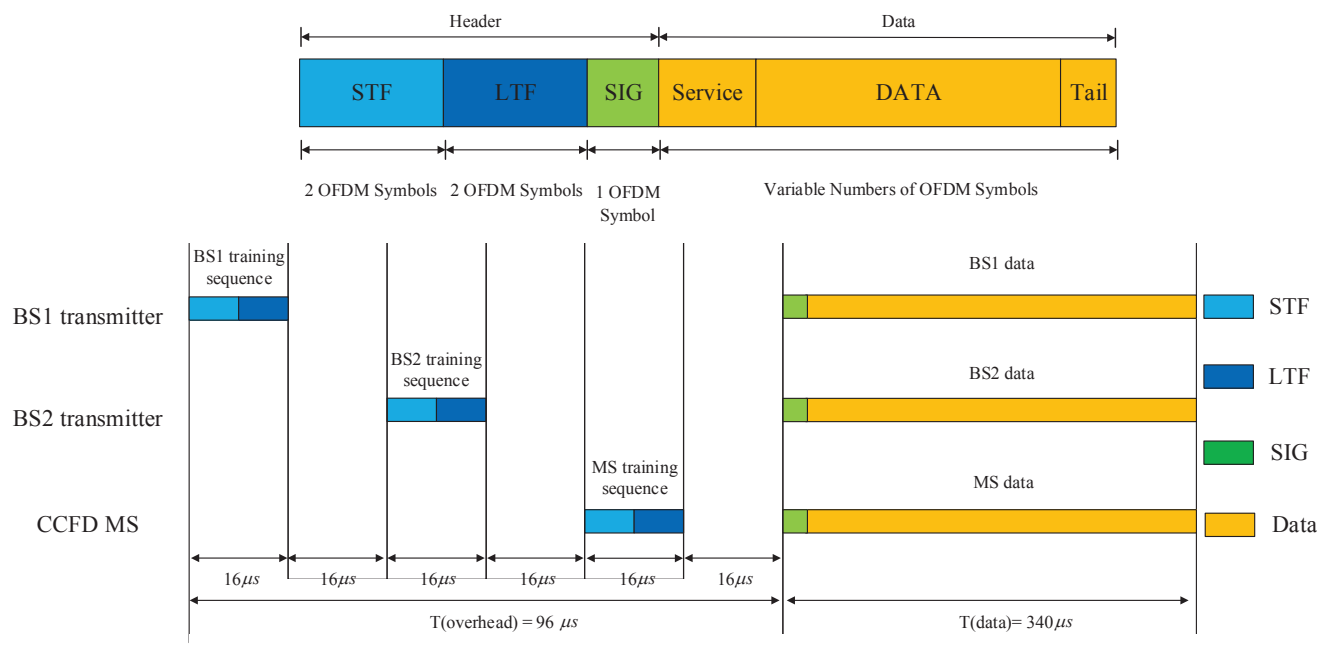

(a)
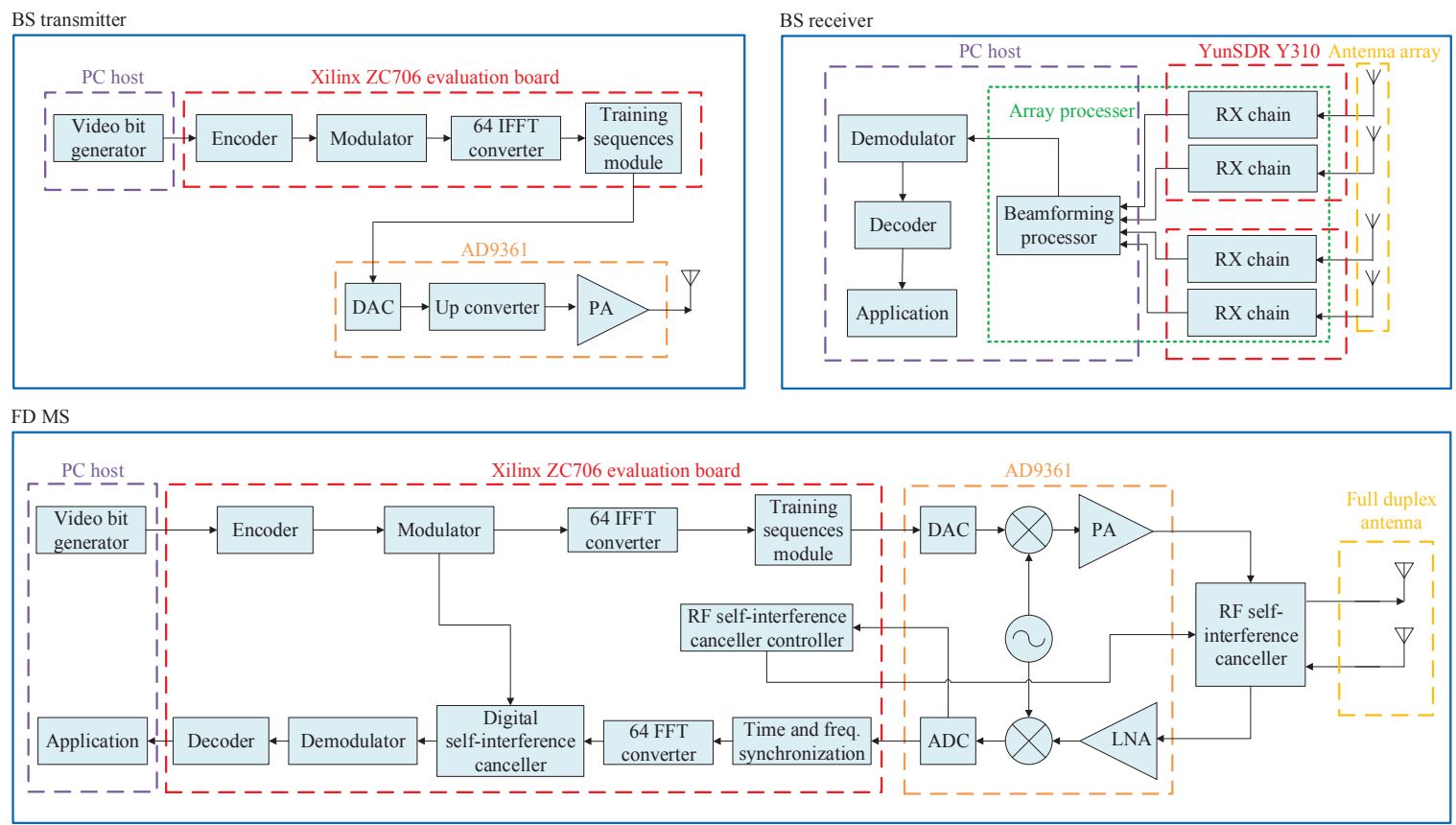

(b)

Figure 3. (a) Designed frame structure and frame interaction; (b) Block diagram of the BS transmitter, BS receiver and FD MS.

packeted into the data frame through the processors of the channel coder, modulator, FFT converter and training sequence adder, according to the proposed specifications in the physical layer. Then, the signals are converted to RF by the DAC, sent to the up-converter for allocating the carrier frequency at $2.4 \mathrm{GHz}$ and through the amplifier to the antenna transmission to complete the DL transmission.

The BS receiver receives the UL signals by the 4 antenna elements connected to the array processor, wherein the signal processing is done by two YunSDR boards to carry out the digital beamforming. The outputs of the array processor are taken to the IFFT converter, decoder and demodulator. Finally, the recovered video bits are shown on the PC screen.

As to the FD MS, the hardware construction of the transmitter is similar to that of the BS. The hardware implementation of the MS's receiver adopts the developed RF self-interference canceler to process the received signals from the full duplex antenna. Then, the digital cancellation is carried out on the ZC706 board. Finally, the video is shown by the PC. 


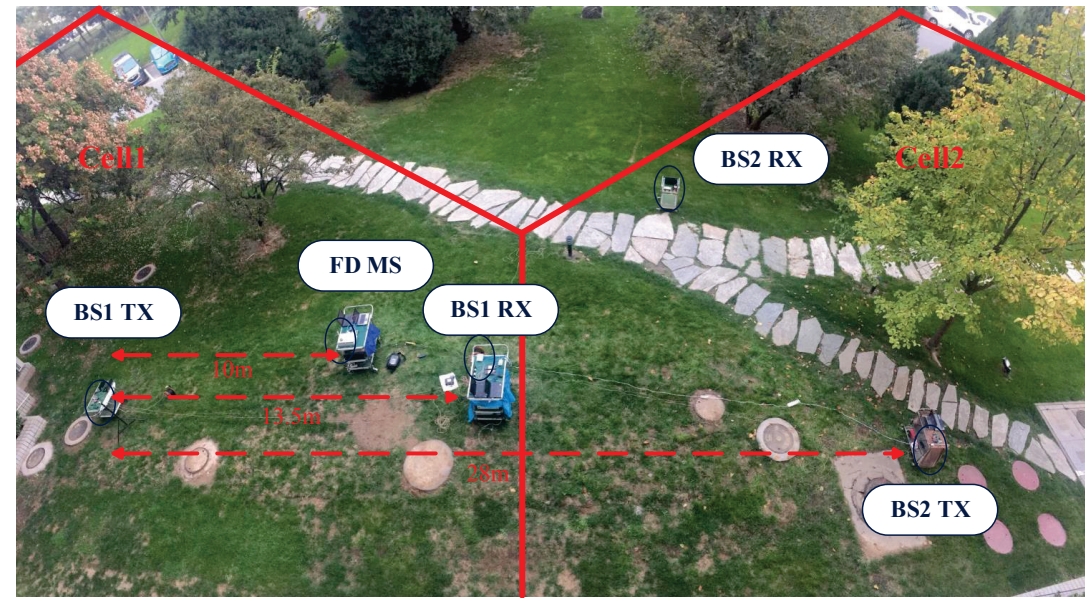

(a)

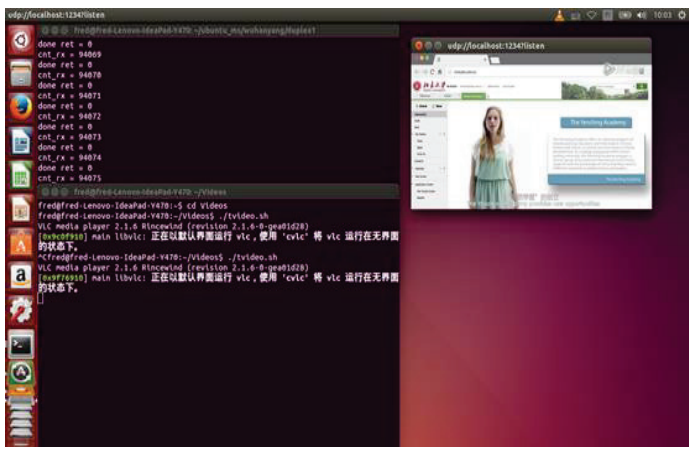

Video on FD MS

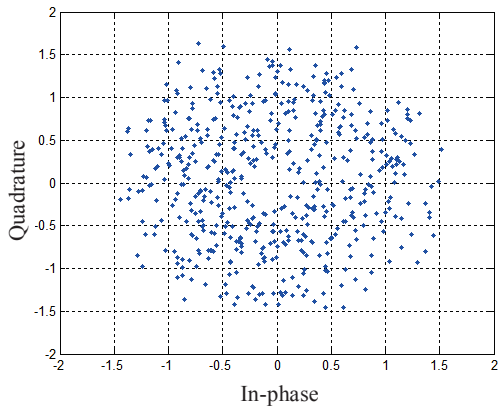

Constellation mapping of one antenna element before beamforming

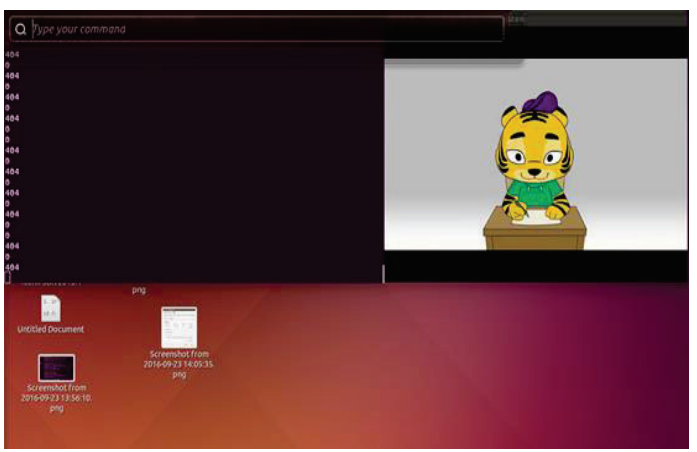

Video on BS RX1

(b)

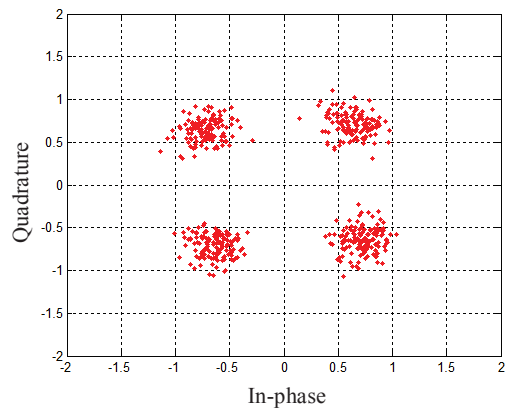

Constellation mapping after interference suppression by beamforming

(c)

Figure 4. (a) Outdoor field test scenario of the demo system; (b) Snapshots of the videos received by FD MS and BS1 RX; (c) Constellation results when the interference suppression at BS1 RX is switched off and switched on, respectively.

\section{Performance Test}

In this section, we present the test results of our prototype system consisting of two cells, each of which covers the area with a radius of 15 meters as shown in the photo taken on the campus of Peking University (see Fig. 4(a)) 


\begin{tabular}{|c|c|}
\hline Item & Value \\
\hline Transmit power of the BS & $0 \mathrm{dBm}$ \\
\hline Transmit power of the FD MS & $-14 \mathrm{dBm}$ \\
\hline Received signal power at the FD MS & $-51 \mathrm{dBm}$ \\
\hline $\begin{array}{l}\text { Power of the M2M interference before self-interference } \\
\text { suppression }\end{array}$ & $-14 \mathrm{dBm}$ \\
\hline $\begin{array}{l}\text { SINR at the FD MS after self-interference } \\
\text { suppression }\end{array}$ & $33 \mathrm{~dB}$ \\
\hline Received signal power at the BS1 RX (one antenna) & $-69 \mathrm{dBm}$ \\
\hline $\begin{array}{l}\text { Power of the B2B interference before beamforming } \\
\text { (one antenna) }\end{array}$ & $-56 \mathrm{dBm}$ \\
\hline SINR at the BS1 RX after beamforming & $15 \mathrm{~dB}$ \\
\hline
\end{tabular}

Table 1. Experimental Settings and Results

The system works on $2.4 \mathrm{GHz}$ with a bandwidth of $20 \mathrm{MHz}$, whereby a conventional rate-1/2 code and QPSK modulation are used in both the BSs' transmitter and that of the FD MS as well. The proposed communication CCFD networking is performed by two active BS-transmitters and one BS receiver. The bi-directional communication is carried out by the FD MS's transmission to the BS receiver array, i.e., BS1 RX for the UL channel and BS1 TX to the MS for the DL channel in one cell. The BS2 TX is delivering the B2B interference.

For the video transmissions, we use a DATA field of 500 bytes to the three transmitters, i.e., the two BS transmitters and the MS transmitter. All sub-carriers of OFDM are filled out by the random information symbols.

The experiments start from setting the transmit powers of each BS-transmitter at $0 \mathrm{dBm}$ and, then, measuring the total power of $\mathrm{B} 2 \mathrm{~B}$ interference by using one antenna at the position of BS1 RX. The received power is found to be $-56 \mathrm{dBm}$. Then, we switch off the BSs' transmissions and set the transmission power of the FD MS at $-14 \mathrm{dBm}$. The received power at the BS1 RX is found to be $-69 \mathrm{dBm}$. The results are listed in Table 1. Finally, the field trials take place for bi-directional video communication with CCFD networking. In the system operation, we measure the SINRs at the BS1 RX and that at the MS. The values are found to be $15 \mathrm{~dB}$ and $33 \mathrm{~dB}$, respectively, as shown in Table 1.

In the field trial, two received videos are demonstrated in the two PC screens of the BS1 receiver and the FD MS as shown in Fig. 4(b). We can see that the video plays are of high quality. The tests also include the investigation of the mobility of the FD MS, where we find that low speed does not affect the quality of the video-transmission at either the receiver of BS1 or that of the FD MS, while high speed motion can distort the two received videos. However, when the FD MS stops its movement, the two videos return quickly to good quality. This indicates the rapid convergence of the algorithm in dealing with the duplex interference.

To show the effectiveness of the interference suppression at the BS receiver, we compare the QPSK constellations in Fig. 4(c), where the constellation contaminated by the B2B interference is shown in the left side and that with the beamforming in the right side. It is found that the points of constellation on the left side are widely spread so that QPSK cannot be identified, while the results on the right side are clearer with the QPSK-profile at the error vector magnitude $(\mathrm{EVM})$ around 5\%. In addition, we investigate the influence of the MSs mobility on the performance, and find the quite similar video qualities at low mobility. Generally, the video performance demonstrates the feasibility of the bi-directional communication over UL and DL channels and confirms this proposed CCFD networking solution. This can provide a practical reference to the application in mobile systems and, at least, to that of small cells.

Finally, we compared the video quality of the CCFD system with that of the half duplex system by switching off the transmission of the DL/UL channel in the prototype. It is observed that the EVM of the constellation in the half duplex system is around 4\%, which is slightly better than that of CCFD.

\section{Vi. Conclusions And Future Directions}

In this paper, the CCFD networking method has been introduced for showing the efficiency of suppressing full duplex interference in a two-cell network. The B2B interference is suppressed by the beamforming method, while the M2M interference by the self-interference canceler. The system has been explained in terms of hardware components and device construction in detail. The experimental results have been presented for showing the capabilities of FD interference nullification and the video-performance is shown for the bidirection communication over UL- and DL channels in the manner of CCFD with very good quality.

For the considerations on further work of mobilenetworking, the potential of combining CCFD with MIMO should be studied for gaining spectral efficiency more effectively, especially in situations involving large-scale antennas. Additionally, the self interference cancellation should be studied for the possible application to satellite communications, where the spectrum is even more scarce in comparison with that of cellular networks. In general, sophisticated techniques would be required in the RF/antenna- and baseband signal processing to guarantee the high quality of communications with doubled spectral efficiency.

\section{ACKNOWLEDGMENT}

The authors would like to thank Dr. Yunzhou Li from Tsinghua University and Dr. Tao Wang from Peking University for their contributions in software and hardware implementations related to this research. 


\section{REFERENCES}

[1] B. Jiao and W. C. Y. Lee, "The interference cancellation method for co-frequency and co-time slot duplex," China Patent 200610113054.4, 2006.

[2] Z. Zhang, X. Chai, K. Long, A. V. Vasilakos and L. Hanzo, "Full duplex techniques for 5G networks: Selfinterference cancellation, protocol design, and relay selection," IEEE Communications Magazine, vol. 53, no. 5, May 2015, pp. 128-137.

[3] K. E. Kolodziej, B. T. Perry and J. S. Herd, "Inband full-duplex technology: Techniques and systems survey," IEEE Transactions on Microwave Theory and Techniques, to appear, 2019.

[4] M. Amjad, F. Akhtar, M. H. Rehmani, M. Reisslein and T. Umer, "Full-duplex communication in cognitive radio networks: A survey," IEEE Communications Surveys \& Tutorials, vol. 19, no. 4, pp. 2158-2191, Fourth quarter 2017.

[5] G. Liu, F. R. Yu, H. Ji, V. C. M. Leung and X. Li, "Inband full-duplex relaying: A survey, research Issues and challenges," IEEE Communications Surveys \& Tutorials, vol. 17, no. 2, pp. 500-524, Second quarter 2015.

[6] S. Huberman and T. Le-Ngoc, "MIMO full-duplex precoding: A joint beamforming and self-interference cancellation structure," IEEE Transactions on Wireless Communications, vol. 14, no. 4, pp. 2205-2217, April 2015.

[7] S. Goyal, P. Liu, S. S. Panwar, R. A. Difazio, R. Yang and E. Bala, "Full duplex cellular systems: Will doubling interference prevent doubling capacity?" IEEE Communications Magazine, vol. 53, no. 5, May 2015, pp. 121-127.

[8] R. Li, Y. Chen, G. Y. Li and G. Liu, "Full-duplex cellular networks," IEEE Communications Magazine, vol. 55, no. 4, Apr. 2017, pp. 184-191.

[9] B. Jiao, S. Liu, Y. Lei and M. Ma, "A networking solution on uplink channel of co-frequency and co-time system," China Communications, vol. 13, no. Supplement 2, 2016, pp. $183-188$.

[10] Xilinx ZC706 Evalution Board User Guide, http: //www.xilinx.com/support/documentation/boards_ and kits/zc706/ug954-zc706-eval-board-xc7z045-apsoc.pdf, 2014.

[11] AD9361 Data Sheet, http://www.analog.com/media/en/ technical-documentation/data-sheets/AD9361.pdf, 2014.

[12] YunSDR Y310 Data Sheet, http://www.v3t.com.cn/ download/Y310 $\ 20$ Datasheet.pdf, 2015.

[13] A. Sahai, G. Patel, and A. Sabharwal, "Pushing the limits of full-duplex: Design and real-time implementation," arXiv:1107.0607, 2011.

[14] IEEE 802.11 Standard: Wireless LAN Medium Access Control (MAC) and Physical Layer (PHY) Specifications.

[15] H. Wu, T. Wang, J. Chen, S. Liu, S. Tian, S. Lu, M. Ma, L. Song and B. Jiao, "GRT-duplex: A novel SDR platform for full-duplex WiFi," Mobile Networks and Applications, vol. 21, no. 6, pp. 983-993, 2016. 